\title{
Diagnostische Abklärung von Hochdruckerkrankungen
}

\author{
Diagnostic Approach to Patients with Arterial Hypertension
}

Autoren

Institute
Georg Schlieper ${ }^{1}$, Nikolaus Marx² ${ }^{2}$ Jürgen Floege ${ }^{1}$, Karl Mischke ${ }^{2}$

${ }^{1}$ Klinik für Nieren- und Hochdruckkrankheiten, rheumatologische und immunologische Erkrankungen,

RWTH Universitätsklinikum Aachen

${ }^{2}$ Klinik für Kardiologie, Pneumologie, Angiologie und Internistische Intensivmedizin,

RWTH Universitätsklinikum Aachen

\section{Schlüsselwörter}

- arterielle Hypertonie

- essenzielle Hypertonie

- sekundäre Hypertonie

- renovaskuläre Hypertonie

- renoparenchymatöse Hypertonie

- Pseudohypertension

- Hyperaldosteronismus

- Schlafapnoe

Key words

- arterial hypertension

- pseudohypertension

- essential hypertension

- secondary hypertension

- renal/renovascular hypertension

- hyperaldosteronism

- sleep apnea

\section{Bibliografie}

DOI http://dx.doi.org/

10.1055/s-0032-1328486

Aktuel Kardiol 2013; 2: 157-163

(c) Georg Thieme Verlag KG

Stuttgart · New York.

ISSN 2193-5203

\section{Korrespondenzadresse}

PD Dr. Georg Schlieper

Oberarzt

Medizinische Klinik II -

Nephrologie und

Klinische Immunologie

RWTH Universitätsklinikum

Aachen

Pauwelsstr. 30

52074 Aachen

Tel.: 0241-8089532

Fax: 0241-8082446

gschlieper@ukaachen.de

\section{Was ist wichtig?}

- Hintergrund und Bedeutung: Die arterielle Hypertonie hat aufgrund ihrer Häufigkeit und der zum Teil schwerwiegenden Folgeschäden eine große sozioökonomische Bedeutung. Eine korrekte Diagnosestellung und frühzeitige Behandlung sind deshalb sehr wichtig.

- Allgemeine Basisdiagnostik: Dazu gehören neben einer korrekt durchgeführten Blutdruckmessung, einer ausführlichen Anamnese und der körperlichen Untersuchung auch Laborkontrollen und apparative Untersuchungen. Insbesondere sollte auch nach möglichen Endorganschäden gesucht werden.

- Korrekte Blutdruckmessung: Goldstandard ist die Langzeit-Blutdruckmessung. Generell sollte eine Hypertonie erst nach mehrmaliger Einzelmessung in Ruhe diagnostiziert werden. Bei Messung mit einer Oberarmmanschette muss auf die richtige Größe geachtet werden.

- Lebensstilanamnese: Insbesondere sollte hier nach den typischen Risikofaktoren gefragt werden. Dazu gehört neben Bewegungsmangel, Übergewicht und ungesunder Ernährung auch der Alkoholkonsum.

- Diagnostische Suche nach spezifischen Ursachen der Hypertonie:

- Medikamente: Die Frage nach Medikamenten gehört bei der Abklärung der arteriellen Hypertonie zur Basisdiagnostik. Zahlreiche Substanzen können einen Bluthochdruck verursachen oder verstärken.

- Schlafapnoe: Diese ist häufig und wird u. a. durch nächtliche Atemaussetzer und Tagesschläfrigkeit symptomatisch. Zur Abklärung eines Verdachts sollte eine ausführliche Anamnese sowie eine Polygrafie und ggf. eine Polysomnografie durchgeführt werden.

- Endokrine Störungen: Zahlreiche endokrine Störungen können einen Bluthochdruck auslösen. Bei therapierefraktärer Hypertonie sollte insbesondere an einen Hyperaldosteronismus, einen Hyperkortisolismus und ein Phäochromozytom gedacht werden.

- Renoparenchymatöse und renovaskuläre Ursachen: Hinweise auf renoparenchymatöse Ursachen liefert meist schon die Basisdiagnostik (GFR, Proteinurie), bei Verdacht auf Nierenarterienstenose sollte eine dopplersonografische Untersuchung erfolgen.

- Sonstige Ursachen: Weitere seltene Ursachen sind eine Aortenisthmusstenose, übermäßiger Lakritzgenuss und Genmutationen.

- Therapierefraktäre Hypertonie: Bei therapierefraktärer Hypertonie sollte immer auch an eine fehlende Therapieadhärenz als Ursache gedacht werden. Differenzialdiagnostisch sind sekundäre Formen, Pseudo- und Praxishypertonie sowie blutdrucksteigernde Medikamentennebenwirkungen auszuschließen.

\section{Hintergrund}

\section{$\nabla$}

Bluthochdruck stellt eine der häufigsten Ursachen für einen Arztbesuch dar. Je nach Population weisen bis zu 30\% der Bevölkerung erhöhte Blutdruckwerte auf. Das Risiko für kardiovaskuläre Ereignisse steigt mit den systolischen und diastolischen Blutdruckwerten, wobei zusätzliche Faktoren das Risiko weiter erhöhen [1]. Die Kategorien der Blutdruckwerte sind in $\odot$ Tab. 1 dargestellt.

\section{Allgemeine Basisdiagnostik} $\nabla$

Zur Basisdiagnostik bei Verdacht auf arterielle Hypertonie zählen wiederholte Blutdruckmessungen mit korrekter Manschette ( Tab. 2), eine Herzfrequenzbestimmung sowie Anamneseerhebung und die klinische Untersuchung. Darüber hinaus kommen Laboruntersuchungen und apparative Untersuchungen zum Einsatz (siehe $\bigcirc$ Tab. 3 und 4). Die diagnostische Abklärung sollte dabei 
Tab. 1 Definitionen und Klassifikation der Blutdruckwerte $(\mathrm{mmHg})$.

\begin{tabular}{|c|c|c|}
\hline \multicolumn{3}{|l|}{ A Praxismessung } \\
\hline Kategorie & systolisch & diastolisch \\
\hline optimal & $<120$ & $<80$ \\
\hline normal & $120-129$ & $80-84$ \\
\hline hoch normal & $130-139$ & $85-89$ \\
\hline Grad 1 Hypertonie (leicht) & $140-159$ & $90-99$ \\
\hline $\begin{array}{l}\text { Grad } 2 \text { Hypertonie } \\
\text { (mittelschwer) }\end{array}$ & $160-179$ & $100-109$ \\
\hline Grad 3 Hypertonie (schwer) & $\geq 180$ & $\geq 110$ \\
\hline isolierte systolische Hypertonie & $\geq 140$ & $<90$ \\
\hline \multicolumn{3}{|c|}{ B Normwert unter standardisierter Belastung (Ergometrie) } \\
\hline \multicolumn{3}{|c|}{ bei 100 Watt systolisch 180 bis max. $200 \mathrm{mmHg}$} \\
\hline \multicolumn{3}{|c|}{ C Normwerte Blutdruck-Selbstmessung } \\
\hline \multirow{2}{*}{\multicolumn{3}{|c|}{$\begin{array}{l}\text { 135/85 mmHg entspricht der Grenze von } 140 / 90 \mathrm{mmHg} \text { in der Praxis } \\
\text { D Ambulante Blutdruck-Langzeitmessung (ABDM): Normwerte und } \\
\text { Definitionen }\end{array}$}} \\
\hline & & \\
\hline & Mittelwerte & $\begin{array}{l}\text { Standard- } \\
\text { abweichung }\end{array}$ \\
\hline Tagesmittelwert & $<135 / 85 \mathrm{mmHg}$ & $<12 / 10 \mathrm{mmHg}$ \\
\hline Nachtmittelwert & $<120 / 75 \mathrm{mmHg}$ & $<14 / 10 \mathrm{mmHg}$ \\
\hline 24-h-Mittelwert & $<130 / 80 \mathrm{mmHg}$ & \\
\hline \multicolumn{3}{|c|}{ E Nachtabsenkung im Vergleich zum Tagesmittelwert } \\
\hline Dipper & $10-20 \%$ & \\
\hline Non-dipper & $<10 \%$ & \\
\hline Inverted dipper & $<0 \%$ & \\
\hline Extreme dipper & $>20 \%$ & \\
\hline
\end{tabular}

Tab. 2 Empfohlene Größe der Blutdruckmanschette in Abhängig vom Armumfang [13].

\begin{tabular}{|l|l|}
\hline $\begin{array}{l}\text { Armumfang } \\
22 \text { bis } 26 \mathrm{~cm}\end{array}$ & $\begin{array}{l}\text { Manschettengröße } \\
12 \times 22 \mathrm{~cm}\end{array}$ \\
\hline 27 bis $34 \mathrm{~cm}$ & $16 \times 30 \mathrm{~cm}$ \\
\hline 35 bis $44 \mathrm{~cm}$ & $16 \times 36 \mathrm{~cm}$ \\
\hline 45 bis $52 \mathrm{~cm}$ & $16 \times 42 \mathrm{~cm}$ \\
\hline
\end{tabular}

umso intensiver sein, je jünger der Patient und je höher der Blutdruck ist bzw. je schneller sich die Hypertonie entwickelt hat.

\section{Blutdruckmessung}

Konkretes Vorgehen. Der Blutdruck weist eine spontane Variation zwischen Tag und Nacht, aber auch zwischen Monaten und Jahreszeiten auf. Somit sollte die Diagnose einer arteriellen Hypertonie auf mehreren Blutdruckmessungen beruhen [2]; in der Regel wird sie durch mindestens 2 Blutdruckmessungen bei einer Visite bzw. durch mindestens 2 bis 3 Arztbesuche gestellt. Bei stark erhöhten Blutdruckwerten und schon bestehenden hypertensiven Organschäden ist evtl. 1 Arztbesuch ausreichend. Initial sollte immer ein Seitenvergleich durch Messung an beiden Armen erfolgen.

Die Blutdruckmessung kann in der Praxis, im Krankenhaus oder zu Hause sowie manuell oder automatisch erfolgen. Bei Messung am Oberarm muss darauf geachtet werden, dass die Manschette die korrekte Größe hat ( Tab. 2), alternativ kann auch am Handgelenk gemessen werden, wobei bei der Handgelenksmessung mehr Fehler (z.B. Messung nicht in Höhe des Herzens) auftreten können. Die Messung sollte in Ruhe, d.h. optimalerweise nach 5minütiger Ruhephase in einem ruhigen Raum erfolgen. Es ist darauf zu achten, dass sich die Blutdruckmanschette auf Herzhöhe befindet. Bei einer reproduzierbaren Blutdruckdifferenz zwischen beiden Armen von $>10-15 \%$ sollte - neben einer Ursachenklärung - immer am Arm mit den höheren Werten gemes- sen werden. Ein exakter Seitenvergleich ist nur mit der simultanen Messung (an beiden Armen gleichzeitig) möglich.

Bei Herzrhythmusstörungen sollte eine Mittelwertbildung erfolgen. Bei der auskultatorischen Blutdruckmessung erfolgt die Bestimmung über Auftreten und Verschwinden der Korotkoff-Geräusche, während automatische Blutdruckmessgeräte fast alle oszillometrisch arbeiten. Beide Methoden sind gut etabliert, es wird die Verwendung von validierten Geräten mit regelmäßiger Kalibrierung und Training empfohlen. Bei älteren Patienten und Diabetikern erscheint es sinnvoll, den Blutdruck zusätzlich zu der Messung im Sitzen im Stehen nach 1 und 5 Minuten zu messen (Hypotonie?). Die häusliche Blutdruckmessung ist in ihrer Wertigkeit wichtiger als die in der Praxis oder im Krankenhaus erfolgte Blutdruckmessung, da eine bessere Korrelation zu kardiovaskulären Ereignissen und Endorganschäden besteht und zusätzlich Effekte eines Weißkittelhypertonus nicht auftreten.

Langzeitblutdruckmessung. Goldstandard der Blutdruckmessung ist die ambulante Langzeitmessung über 24 Stunden (ABDM). Durch die relativ hohe Messdichte von ca. 70 Messungen über 24 Stunden (tagsüber alle 15 Minuten und nachts alle 30 Minuten) steigt die Sicherheit bei der Bewertung des wahren Blutdruckniveaus im Vergleich zu Einzelmessungen drastisch an. Mit der ABDM können u. a. erfasst werden:

- die durchschnittliche Blutdruckhöhe am Tag und in der Nacht

- Blutdruckschwankungen und die Blutdruckvariabilität (z.B. Standardabweichung)

- Fehlen oder Verminderung der normalen Blutdrucksenkung in der Nacht oder außergewöhnliche Blutdruckanstiege in der Nacht oder in den Morgenstunden [3].

Eine verminderte Nachtabsenkung des Blutdrucks bzw. ein Anstieg während des Schlafes ist häufig bei sekundären Hochdruckformen ( Tab. 5) oder schweren hypertensiven Organschäden zu finden.

Die alleinige Praxismessung führt in ca. 30\% zu einer falschen Charakterisierung der Patienten bzw. der Blutdrucksituation (Praxishypertonie bzw. Praxisnormotonie oder maskierte Hypertonie) [4]. Im Vergleich zur Praxismessung in Ruhe hat die Messung des Belastungsblutdrucks während der Ergometrie den Vorteil der Erfassung des Blutdruckverhaltens unter standardisierter, reproduzierbarer körperlicher Belastung sowie der Minimierung psychischer Einflüsse.

Pseudohypertonie. Traditionell beschreibt die Pseudohypertonie falsch erhöht gemessene Blutdruckwerte. Sie tritt besonders bei verkalkten Gefäßen auf (Mediasklerose), die bei diabetischen und niereninsuffizienten Patienten häufig sind. Aufgrund der fehlenden Kompressibilität des steifen Gefäßes wird der Blutdruck höher gemessen als er eigentlich (d.h. intraarteriell) ist. Hierzu hat es in den letzten 2 Jahrzehnten wenig belastbare neue Forschungsdaten gegeben, sodass bei inzwischen niedrigeren Zielblutdruckwerten die Bedeutung der Pseudohypertonie und auch die Verwendung des Begriffs der Pseudohypertonie kritisch hinterfragt wird [5].

\section{Kurzgefasst}

Eine korrekte Blutdruckmessung (z. B. initial beidseitige Messung, Nutzung korrekter Manschetten, etc.) ist wichtig. Die Diagnose einer arteriellen Hypertonie kann in der Regel erst nach mehrmaliger Messung, optimalerweise unter häuslichen Bedingungen erfolgen. Die Langzeit-Blutdruckmessung ist der Goldstandard zur Erfassung einer manifesten Hypertonie und zur Charakterisierung des Tag-Nacht-Rhythmus. 
Tab. 3 Basishypertonieabklärung: Anamnese und Labor.

\begin{tabular}{|c|c|}
\hline \multicolumn{2}{|l|}{ Anamnese } \\
\hline $\begin{array}{l}\text { Nierenerkankung } \\
\text { (auch familiär) }\end{array}$ & Zystennieren \\
\hline Medikamente/Drogen & $\begin{array}{l}\text { nicht steroidale Antiphlogistika, } \\
\text { einschließlich Aspirin } \\
\text { - COX2-Inhibitoren } \\
\text { - Kortikosteroide } \\
\text { - Sympathomimetika (Diätetika) } \\
\text { - orale Kontrazeptiva } \\
\text { - Zyklosporin } \\
\text { - Erythropoetin (hohe Dosierungen) } \\
\text { - Alkohol } \\
\text { - Kokain } \\
\text { - exzessive Lakritzmengen } \\
\text { Stimulanzien, z. B. Amphetamine } \\
\text { oder Methylphenidat }\end{array}$ \\
\hline Hinweis für Phäochromozytom & $\begin{array}{l}\text { Blutdruckkrisen mit Palpitation, } \\
\text { Schwitzen, Kopfschmerzen }\end{array}$ \\
\hline $\begin{array}{l}\text { Hinweis für Hyperaldo- } \\
\text { steronismus }\end{array}$ & Muskelschwäche, (Tetanie?) \\
\hline kardiovaskuläre Risikofaktoren & $\begin{array}{l}\text { Diabetes, Nikotin, BMI, Ernährung, } \\
\text { Kochsalz, Bewegung, etc. }\end{array}$ \\
\hline $\begin{array}{l}\text { (Fremd-)Anamnese } \\
\text { bzgl. Schlafapnoe }\end{array}$ & $\begin{array}{l}\text { Tagesschlärrigkeit, „Schnarcht Ihr Part- } \\
\text { ner“, „Hat Ihr Partner Atemaussetzer?“ }\end{array}$ \\
\hline zerebrale Symptomatik & $\begin{array}{l}\text { Kopfschmerz, Schwindel, Sehstörung, } \\
\text { TIA, andere Defizite }\end{array}$ \\
\hline Herzsymptomatik & Angina, Palpitationen, Dyspnoe, Ödeme \\
\hline Nierensymptomatik & $\begin{array}{l}\text { Ödeme, Durst, Polyurie, Nykturie, } \\
\text { Hämaturie }\end{array}$ \\
\hline periphere Gefäße & Claudicatio, kalte Extremitäten \\
\hline bisherige Therapie & Medikation und Nebenwirkungen \\
\hline weitere Anamnese & $\begin{array}{l}\text { Familien- und soziale Anamnese (fami- } \\
\text { liäre Hypertonie/genetische Disposition) }\end{array}$ \\
\hline \multicolumn{2}{|l|}{ Laborparameter } \\
\hline Metabolismus & $\begin{array}{l}\text { Glukose i.S. } \\
\text { Blutfette i.S. (Triglyzeride, Gesamt-, } \\
\text { HDL- und LDL-Cholesterin) } \\
\text { - Harnsäure i.S. }\end{array}$ \\
\hline Niere & $\begin{array}{l}\text { Kreatinin i.S. } \\
\text { eGFR (MDRD, CKD-Epi oder Cockroft } \\
\text { Gault) } \\
\text { Urinstatus (Proteinurie, Mikro- } \\
\text { hämaturie, Mikroalbuminurie) }\end{array}$ \\
\hline Blutbild & Hämoglobin \\
\hline Elektrolyte & Kalium i.S. \\
\hline
\end{tabular}

Labor

$\mathrm{Zu}$ jeder Basisabklärung gehört eine Untersuchung von Laborparametern (siehe $\bigcirc$ Tab.3). Die Analyse von Retentionswerten und Elektrolyten kann Hinweise auf eine sekundäre Hypertonie liefern. Die alleinige Bestimmung des Serumkreatinins genügt nicht, hier sollte aufgrund des kreatininblinden Bereichs eine Bestimmung der glomerulären Filtrationsrate mittels Formel (MDRD, CKD-Epi oder Cockroft Gault) erfolgen. Die Untersuchung von metabolischen Parametern dient der Erkennung von zusätzlichen kardiovaskulären Risikofaktoren.

Kurzgefasst

Die Bestimmung von Laborparametern, wie Kreatinin und Elektrolyten, gehört zur Basisdiagnostik.
Tab. 4 Apparative Diagnostik zur Detektion von Endorganschäden.

\begin{tabular}{ll}
\hline EKG & $\begin{array}{l}\text { Sokolow-Lyon-Index? Cornell-Voltage-QRS- } \\
\text { Produkt? Endstreckenveränderungen? }\end{array}$ \\
\hline Echokardiografie & $\begin{array}{l}\text { Septumdicke? Massenindex? Funktion? } \\
\text { Vitium? }\end{array}$ \\
\hline Abdomensonografie & Aortenaneurysma? Schrumpfnieren? \\
\hline Knöchel-Arm-Index & pAVK? \\
\hline Karotisdoppler & Intima-Media? Plaques? \\
\hline Funduskopie & Retinopathie? \\
\hline $\begin{array}{l}\text { quantitative Proteinurie/ } \\
\text { (Mikro-)Albuminurie }\end{array}$ & Nephropathie? \\
\hline
\end{tabular}

\section{Diagnostik von Sekundärschäden}

Eine Abklärung auf Endorganschäden (siehe $\bigcirc$ Tab. 4) wird empfohlen, da bereits ein subklinischer Endorganschaden einen weiteren kardiovaskulären Risikofaktor darstellt. In retrospektiven Studien war die effiziente Behandlung einer Mikroalbuminurie oder linksventrikulären Hypertrophie mit einer Prognoseverbesserung vergesellschaftet. Bei sehr hohen Blutdruckwerten ist die Untersuchung des Augenhintergrunds sinnvoll. Bez. zerebraler Folgeschäden sollte eine Karotis-Doppler-Untersuchung durchgeführt werden und bei klinischem Verdacht kann je nach zerebraler Symptomatik eine kraniale Bildgebung mittels CT oder MRT oder auch eine kognitive Testung sinnvoll sein.

\section{Kurzgefasst}

Die Detektion von Endorganschäden, insbesondere an Herz, Nieren, Augen und Gehirn, gehört zur Basisdiagnostik.

\section{Lebensstilanamnese}

Eine Abfrage des Lebensstils gehört zur Routinediagnostik bei arterieller Hypertonie. Bei der Anamnese sollten insbesondere Essgewohnheiten (vor allem Fett- und Salzzufuhr), Gewicht, Sport, Alkoholkonsum und Nikotinabusus abgefragt werden.

Bewegungsmangel. Bewegungsmangel ist mit einem Risiko für arterielle Hypertonie und Übergewicht verbunden. Dynamisches Ausdauertraining reduziert Blutdruck, Gewicht und Bauchumfang bei Steigerung der Insulinsensitivität und des HDL-Cholesterins. Patienten mit sitzender Tätigkeit sollte deshalb ein tägliches Training mit moderater Intensität über ca. 30-45 min empfohlen werden.

Übergewicht. Das Gewicht ist mit dem Blutdruck assoziiert. Übermäßiges Körperfett prädisponiert zu arterieller Hypertonie. Neben dem aktuellen Gewicht (und dem Body-Mass-Index) sollte auch eine Gewichtsveränderung seit dem frühen Erwachsenenalter abgefragt werden.

Salzkonsum und weitere Essgewohnheiten. Essenzielle arterielle Hypertonie wird überwiegend in Gesellschaften mit hoher Natriumaufnahme (>100 mmol/d, d. h. $>5,84 \mathrm{~g} / \mathrm{d}$ ) beobachtet [7]. Eine exzessive Natriumchloridzufuhr kann Ursache für eine therapierefraktäre arterielle Hypertonie sein. Eine niedrige Kaliumzufuhr verstärkt die ungünstige Wirkung einer hohen Natriumaufnahme. Prinzipiell muss in Deutschland bei den meisten Patienten von einer zu hohen täglichen Salzzufuhr ausgegangen werden. Ebenso wird ein hoher Konsum von gezuckerten Getränken unabhängig von einer Gewichtszunahme mit einer arteriellen Hypertonie in Verbindung gebracht, möglicherweise bedingt durch Sympathikusaktivierung, Reduktion der $\mathrm{Na}^{+}$-Ausscheidung und Erhöhung der Harnsäure mit Reduktion von endothelialem Stickstoffmonoxid und/oder Aktivierung des Renin-Angiotensin- 
Systems. Kaffee führt akut zu einem Blutdruckanstieg, die Datenlage bezüglich einer Assoziation von regelmäßigem Kaffeekonsum und arterieller Hypertonie ist aber uneinheitlich.

Alkoholkonsum. Zwischen Alkoholkonsum und Prävalenz einer arteriellen Hypertonie besteht eine lineare Beziehung [8]. Bei der diagnostischen Abklärung der arteriellen Hypertonie ist zu beachten, dass ein akuter Entzug bei Patienten mit hohem Alkoholkonsum zu arterieller Hypertonie führen kann. So können etwa hohe Blutdruckwerte am Wochenbeginn auf Alkoholentzug nach exzessivem Konsum am Wochenende beruhen.

Eine Lebensstilmodifikation gehört immer zur Basistherapie bei arterieller Hypertonie. Die wichtigsten Faktoren sind dabei sportliche Betätigung, Gewichtsnormalisierung, salzarme Kost und weitere diätetische Maßnahmen sowie Verzicht auf Rauchen und Einschränkung des Alkoholkonsums. Wenngleich eine Beendigung des Rauchens nicht den Blutdruck senkt, führt sie zu einer deutlichen Reduktion des kardiovaskulären Risikos und sollte deshalb allen Patienten empfohlen werden. Ziel einer Lebensstilmodifikation ist neben einer Blutdruckabsenkung auch eine Reduktion kardiovaskulärer Risikofaktoren.

Eine kaliumreiche Diät mit einem hohen Anteil an Obst, Gemüse und Fisch sowie Reduktion von Cholesterin, gesättigter Fette und gezuckerter Getränke senken den Blutdruck und reduzieren das kardiovaskuläre Risiko.

\section{Kurzgefasst}

Der Lebensstil beeinflusst den Blutdruck. Risikofaktoren für die Entwicklung oder das Vorliegen einer arteriellen Hypertonie sind dabei insbesondere Bewegungsmangel, Übergewicht, ungesunde Ernährung und Alkoholkonsum. Nach diesen Faktoren sollte deshalb bei der Anamnese immer gefragt werden.

\section{Ursachenspezifische Anamnese und Diagnostik} $\nabla$

\section{Medikamentös induzierte Hypertonie}

Verschiedene Medikamente führen als Nebenwirkung zu einer Erhöhung des arteriellen Blutdrucks. Insbesondere wird dies beobachtet bei der Verwendung nicht steroidaler Antiphlogistika einschließlich COX2-Inhibitoren. Besonders bei älteren Patienten konnte gezeigt werden, dass die Verwendung nicht steroidaler Antiphlogistika zu einer Erhöhung des systolischen Blutdrucks führt. Eine hierzu durchgeführte Metaanalyse zeigte, dass nicht steroidale Antiphlogistika den Blutdruck um bis zu $5 \mathrm{mmHg}$ erhöhen. Jedoch steigern nicht alle nicht steroidalen Antiphlogistika den Blutdruck in gleichem Maße. Klinische Studien legen nahe, dass z.B. Rofecoxib den systolischen Blutdruck im Vergleich zu Celecoxib deutlicher erhöht. Die zugrunde liegenden Mechanismen dieser Blutdruckerhöhung sind nicht in Gänze verstanden. Ein erhöhter vaskulärer Widerstand über eine erhöhte Endothelin-1-Synthese oder Störungen im Arachidonsäuremechanismus (Verminderung der vasodilatierenden Prostazyklinproduktion der Gefäßwand) werden hier ursächlich diskutiert. In diesem Zusammenhang sollte aber erwähnt werden, dass auch ein Schmerzstimulus selbst erhöhte Blutdruckwerte induzieren kann.

Dass Glukokortikoide zu einer Blutdruckerhöhung führen, ist seit Langem bekannt, wobei der Effekt bei älteren Patienten im Vergleich zu jüngeren Patienten häufiger beobachtet wird. Unter einer oralen Glukokortikoidtherapie kann es binnen 24 Stunden zu einer systolischen Blutdruckerhöhung bis über $10 \mathrm{mmHg}$ kommen. Insbesondere die Mineralokortikoid-Komponente von Kortikoiden, die zu einer Expansion von Natrium und Blutvolumen führt, verursacht hier die Blutdruckerhöhung.

Bei postmenopausalen Frauen kann die Therapie mit Östradiol zu einer Blutdruckerhöhung führen. Dies betrifft jedoch vor allem jüngere postmenopausale Frauen.

Weitere Substanzen, die zur Blutdruckerhöhung oder zur Verminderung der Effektivität antihypertensiver Medikamente führen, sind Amphetamine, orale Kontrazeptiva, Zyklosporin, Erythropoetin sowie trizyklische Antidepressiva ( $\bullet$ Tab. 3).

Prinzipiell gilt, dass eine differenzierte Anamnese in Bezug auf die vom Patienten eingenommenen Medikamente und Genussmittel erhoben werden sollte. Auch auf den Alkoholgenuss, den Genuss von gezuckerten Getränken, den Genuss von Lakritze und bestimmten Medikamenten, die zur Gewichtsreduktion eingesetzt werden, sollte fokussiert werden.

\section{Kurzgefasst}

Zahlreiche Medikamente können zu einer Blutdruckerhöhung führen. Die Medikamentenanamnese gehört deshalb zur Basisdiagnostik. Eine besondere Rolle spielen dabei NSAR und Glukokortikoide, aber auch Hormonersatztherapie, Kontrazeptiva, Psychopharmaka, Immunsuppressiva und viele andere Substanzen können eine arterielle Hypertonie auslösen oder verstärken.

\section{Schlafapnoe}

Trotz einiger Schwierigkeiten bei der Beurteilung eines kausalen Zusammenhangs zwischen obstruktiver Schlafapnoe (OSA) und arterieller Hypertonie (z.B. aufgrund gemeinsamer Risikofaktoren), gilt die obstruktive Schlafapnoe als eine häufige Ursache für eine sekundäre Hypertonie. Durch intermittierende Hypoxie und Schlafunterbrechung kommt es akut zu einer Sympathikusaktivierung mit Hypertonie und chronisch zu einer Änderung des Gefäßwiderstands, arterieller Hypertonie und endothelialer Dysfunktion. Die obstruktive Schlafapnoe ist häufig und findet sich bei etwa jedem 5. Erwachsenen, ein obstruktives Schlafapnoesyndrom mit Beeinträchtigung des Patienten am Tage tritt bei etwa einem von 20 Erwachsenen auf [9].

Eine obstruktive Schlafapnoe erhöht in Abhängigkeit ihres Schweregrads das Risiko, eine arterielle Hypertonie zu entwickeln [10]. Gleichzeitig kann eine CPAP-Beatmung dieses Risiko reduzieren [10].

Aufgrund der hohen Prävalenz der OSA sollte bei Patienten mit arterieller Hypertonie unter anderem an eine Schlafapnoe gedacht werden. Insbesondere bei Patienten mit therapierefraktärer Hypertonie und bei Patienten mit fehlender Nachtabsenkung in der Langzeit-Blutdruckmessung ist eine Schlafapnoeabklärung wichtig.

Zur Diagnostik der Schlafapnoe werden Anamnese und Fremdanamnese, ein standardisierter Fragebogen, die klinische Untersuchung und die ambulante Polygrafie herangezogen. $\mathrm{Zu}$ den Leitsymptomen der obstruktiven Schlafapnoe zählen lautes und unregelmäßiges Schnarchen mit Atemstillständen in der Fremdanamnese sowie eine erhöhte Tagesschläfrigkeit mit Einschlafneigung. Weitere Zeichen können Gereiztheit, eingeschränkte Konzentrationsfähigkeit, gehäufte Verkehrsunfälle, Erstickungsalbträume und morgendliche Kopfschmerzen sein. Zur weiteren Abklärung sollten dann standardisierte Fragebögen wie etwa die Epworth Sleepiness Scale oder der Berlin-Fragebogen eingesetzt 
werden. Zum Schlafapnoe-Screening erfolgen ambulant durchgeführte Polygrafien. Zeigt sich hier ein pathologischer Apnoe-/ Hypopnoe-Index (AHI), sollte eine Polysomnografie im Schlaflabor durchgeführt werden bzw. bei schwerer Schlafapnoe direkt mit der Therapie begonnen werden. Anhand des AHI kann eine Einteilung der Schlafapnoe in leicht (AHI 6-14), mittel (AHI 1529) und schwer $(\mathrm{AHI} \geq 30)$ erfolgen.

\section{Kurzgefasst}

Bei Verdacht auf sekundäre Hypertonie sollte eine Schlafapnoe ausgeschlossen werden. Diese ist bei Erwachsenen häufig (bis ca. 20\%) und kann einen Bluthochdruck auslösen oder verstärken. Anamnestisch deuten unter anderem unregelmäßiges Schnarchen, nächtliche Atemstillstände (Fremdanamnese), Tagesschläfrigkeit, morgendliche Kopfschmerzen und Einschlafneigung auf die Schlafapnoe hin. Zur diagnostischen Abklärung sollte eine Polygrafie und ggf. eine Polysomnografie durchgeführt werden.

\section{Endokrine Ursachen}

Die Bestimmung endokrinologischer Parameter gehört nicht zur Basisdiagnostik bei arterieller Hypertonie [2]. Typischerweise werden endokrine Hochdruckursachen daher spät diagnostiziert, obwohl gerade in solchen Fällen eine spezifische Therapie zur Normalisierung des Blutdrucks führen kann. Während eine Vielzahl endokriner Störungen zu einer Blutdruckerhöhung führt (siehe Tab. 5), soll hier nur auf die 3 wichtigsten Ursachen und deren Basisdiagnostik eingegangen werden [11]. Angesichts der oft aufwendigen und kostenintensiven Diagnostik muss durch klinische Befunde und einfache Laboruntersuchungen die „Treffer-Wahrscheinlichkeit“ erhöht werden. Hinweise können sein:

- Hyperaldosteronismus: therapierefraktäre Hypertonie oder mittelschwere Hypertonie mit niedrig-normalem oder erniedrigtem Serumkalium (sofern keine Diuretika eingesetzt werden), positive Familienanamnese, zufälliger Nachweis einer adrenalen Raumforderung („Inzidentalom“)

- Phäochromozytom: krisenhafte Blutdruckanstiege (Cave: nicht selten jedoch auch dauerhaft erhöhter Blutdruck!), Kopfschmerz, Schwitzen, Blässe, Palpitationen

- Hyperkortisolismus: Blickdiagnose! Cushing-Habitus, insbesondere Striae rubrae.

Hyperaldosteronismus. Vor allem bei schweren Hypertonieformen, wie sie in Spezialambulanzen gesehen werden, findet sich bei 4 bis zu 32\% einer therapieresistenten Hypertonie eine mögliche Aldosteronabhängigkeit. Als Suchtest eignet sich die morgendliche Bestimmung von Renin und Aldosteron im Serum nach 10-minütiger Ruhepause. Bei primärem Hyperaldosteronismus ist der Aldosteron-Renin-Quotient angesichts der Suppression des Renins durch das hohe Aldosteron stark erhöht. Aldosteronantagonisten und kaliumsparende Diuretika sollten mehrwöchig vor der Blutabnahme pausiert werden. Auch eine Kochsalzrestriktion und die Gabe von Schleifendiuretika sollte möglichst unterbrochen werden. Die weiterführende Diagnostik umfasst einen Kochsalzbelastungstest sowie Bildgebung und ggf. die seitengetrennte Blutabnahme aus den Nebennieren.

Phäochromozytom. Als Suchtest wird in der Regel die Bestimmung freier Katecholamine (Adrenalin, Noradrenalin) oder Metanephrine (Metanephrin und Normetanephrin) im 24-h-Sammelurin eingesetzt. Zwischen den Blutdruckkrisen kann die Katecholaminausscheidung normal sein, sodass bei hochgradigem Ver-
Tab. 5 Sekundäre Ursachen einer arteriellen Hypertonie.

Schlafapnoe
renovaskulär (atherosklerotische/fibromuskuläre Nierenarterienstenose)
renoparenchymatös
endokrin
- Hyperaldosteronismus, Conn-Syndrom
- Phäochromozytom bzw. multiple endokrine Neoplasia-(MEN-)Syndrome
- Cushing-Syndrom und Hyperkortisolismus
- Hyper- und Hypothyreose
- Akromegalie
Medikamente/Drogen (siehe $\bullet$ Tab. 3 )
Aortenisthmusstenose, monogenetisch, weitere seltene Ursachen

dacht eine Mehrfachsammlung erfolgen sollte - möglichst im Anschluss an eine hypertensive Krise. Sensitiver ist die Bestimmung der Plasma-Metanephrine nach einer 20-minütigen Ruhephase im Liegen; diese Analysemöglichkeit ist zunehmend breiter verfügbar.

Hyperkortisolismus. Als nahezu diagnostisch gleichwertig gelten die Bestimmung des Kortisoltagesprofils im Speichel, die Messung von freiem Kortisol im 24-h-Sammelurin sowie der LowDose-(1 mg-)Dexamethason-Hemmtest. Mindestens 2 dieser Tests sollten positiv ausfallen. Die weiterführende Diagnostik beinhaltet vor allem ACTH-Bestimmungen (Differenzierung adrenaler vs. hypophysärer Morbus Cushing).

\section{Kurzgefasst}

Bei Verdacht auf sekundäre Hypertonie sollte eine endokrinologische Ursache ausgeschlossen werden. Differenzialdiagnostisch müssen vor allem Hyperaldosteronismus, Hyperkortisolismus und Phäochromozytom in Erwägung gezogen werden.

\section{Renale Ursachen}

Renoparenchymatöse Hypertonie. Renoparenchymatöse Ursachen sind für ca. 5-10\% der arteriellen Hypertoniefälle verantwortlich. Neben klinischen Hinweisen (Anamnese, Ödeme, Nykturie u.a.) lenkt in der Regel die Basisdiagnostik, d.h. eine reduzierte eGFR und/oder Auffälligkeiten im Urin-Stix, den Verdacht rasch auf eine renale Genese des Hochdrucks. Als sensitivster Marker gilt die Bestimmung der Mikroalbuminurie, die naturgemäß sowohl Ausdruck einer zugrunde liegenden renalen Erkrankung als auch einer hypertensiven Folgeerkrankung sein kann. Bei fortgeschrittener renaler Beteiligung im Rahmen einer zunächst primären Hypertonie, die nicht adäquat therapiert ist, kann es sogar zur nephrotischen Proteinurie (d.h. > 3,5 g/d) kommen. In solchen Fällen kann oftmals nur eine Nierenbiopsie die „Henne und Ei-Frage“ klären, d.h. die Differenzierung zwischen renaler Ursache der Hypertonie vs. hypertensiver Nephropathie. Renovaskuläre Hypertonie. Schwieriger gestaltet sich die Situation bei renovaskulärer Hypertonie, d.h. ein- oder beidseitiger Nierenarterienstenose. Klinisch fallen evtl. krisenhafte Blutdruckanstiege auf (evtl. mit sog. „flash“-Lungenödem) und in Einzelfällen kann sich ein Strömungsgeräusch im Oberbauch finden. Nicht selten sind Nierenarterienstenosen jedoch komplett asymptomatisch. Ein laborchemisches Verdachtsmoment kann eine nicht erklärte (d.h. nicht diuretikainduzierte oder anders bedingte) Hypokaliämie bieten, da es durch die Aktivierung des Renin-Angiotensin-Aldosteron-Systems zur Kaliurie kommt. In 
unserer Praxis hat sich vor allem die beidseitige dopplersonografische Bestimmung des renalen Widerstandsindexes als Suchmethode bewährt ( $\mathbf{A b b} \mathbf{1}$ ). Sowohl ein einseitiger Widerstandsindex unter 0,45 als auch eine Seitendifferenz über $10 \%$ sind suggestiv für eine Nierenarterienstenose [12]. Bei hochgradigem Verdacht (und therapeutischen Konsequenzen) sollte danach unmittelbar eine Angiografie erfolgen.

\section{Kurzgefasst}

Bei Verdacht auf sekundäre Hypertonie sollte eine renale bzw. renovaskuläre Ursache ausgeschlossen werden. Hinweise auf eine renoparenchymatöse Ursache kann schon die Basisdiagnostik, insbesondere die Bestimmung der GFR und der UrinStix geben. Bei Verdacht auf Nierenarterienstenose sollte zunächst eine Doppler-Sonografie durchgeführt werden.

\section{Seltene Ursachen}

Aortenisthmusstenose. Die Aortenisthmusstenose beruht auf einer postnatalen Schrumpfung von Gewebe des Ductus botalli, das die Aorta umgibt. Sie führt zu einer Hypertonie der oberen Extremitäten mit einer signifikanten Seitendifferenz und zur Hypotonie der unteren Extremitäten mit abgeschwächtem Leistenpuls. Daraus resultieren Symptome wie Kopfschmerzen, kalte Füße mit abgeschwächtem Puls sowie ein Systolikum über dem Aortenareal. Im Röntgenbild des Thorax sind die charakteristischen Rippenusuren nachweisbar. Neben der Diagnostik mittels Echokardiografie kann eine weitere Bildgebung mittels Computertomografie und Magnetresonanztomografie erfolgen. Dabei erlaubt die Magnetresonanztomografie auch eine Fluss- und Gradientenbestimmung. Bei Diagnose einer Aortenisthmusstenose sollte auf assoziierte Anomalien geachtet werden wie etwa eine bikuspide Aortenklappe.

Lakritze. Die in Lakritze (Süßholz) enthaltene Glycyrrhizinsäure greift über eine Hemmung der Steroid-5 $\beta$-Reduktase und der $11 \beta$-Hydroxysteroid-Dehydrogenase 2 in den Steroidstoffwechsel ein und kann so bei exzessivem Genuss zu einer Hypertonie führen. Lakritzhaltige Genussmittel sind insbesondere in den Niederlanden beliebt.

Monogenetische Hypertonieformen. Monogenetische Hypertonieformen führen häufig über eine Aktivierung von Mineralokortikoidrezeptoren und durch überaktive epitheliale Natriumkanäle der Niere zu einer Blutdrucksteigerung. Zu den monogenetischen Hypertonieformen zählt etwa das Liddle-Syndrom („gain-of-function“-Mutation des epithelialen Natriumkanals mit erhöhter renaler Natriumrückresorption).

\section{Kurzgefasst}

Weitere seltene Ursachen für eine sekundäre Hypertonie sind die Aortenisthmusstenose, ein Lakritzabusus und genetische Faktoren.

\section{Therapierefraktäre Hypertonie}

$\nabla$

Die Definition einer resistenten oder therapierefraktären Hypertonie meint in der Regel die fehlende Einstellung des systolischen oder diastolischen Blutdrucks auf den Zielwert, wenn mindestens 3 Antihypertensiva verschrieben wurden, wobei eines davon ein Diuretikum sein sollte [2].

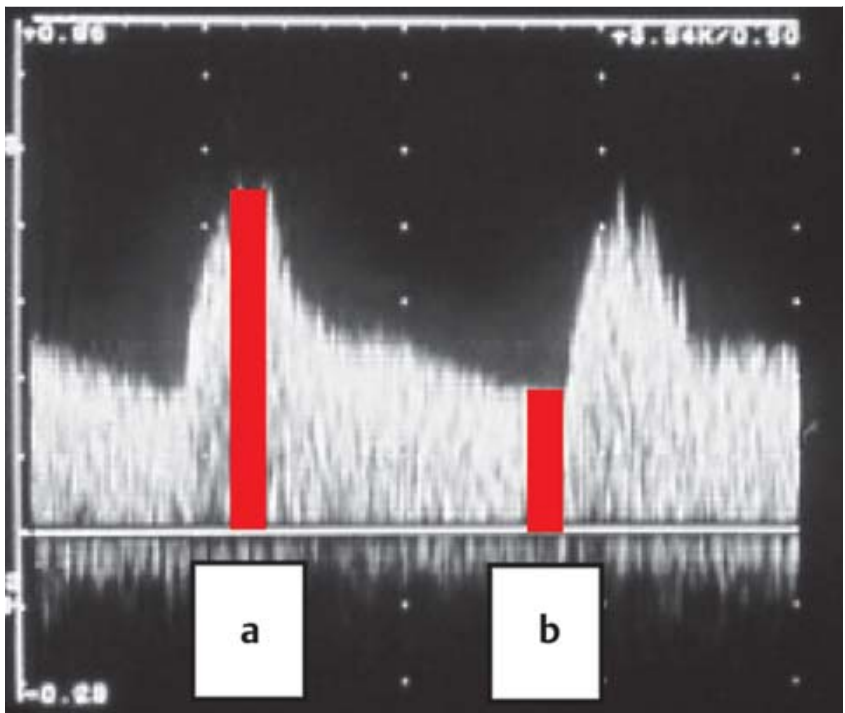

Abb. 1 Widerstandsindex. Der intrarenal gemessene Widerstandsindex, gemessen als Differenz „systolisches Maximum (a) minus diastolisches Minimum (b)“ im Verhältnis zum systolischen Maximum (a) [Formel: $\mathrm{WI}=(\mathrm{a}-\mathrm{b}) / \mathrm{a}]$ kann insbesondere im Seitenvergleich (signifikante Reduktion?) ein Hinweis für eine vorliegende Nierenarterienstenose bieten.

Verwendet man diese Klassifikation, so lag in einer großen Hypertoniestudie (ALLHAT-Studie) die Inzidenz der resistenten Hypertonie bei $15 \%$. Insgesamt kommen verschiedene Ursachen infrage [6]. Eine ganz entscheidende Ursache der insuffizienten Hypertonietherapie ist die ungenügende Adhärenz zur medikamentösen Therapie. Hier konnte gezeigt werden, dass $1 \mathrm{Jahr}$ nach Beginn einer antihypertensiven Monotherapie nur noch ca. $2 / 3$ der Patienten ihr Medikament regelmäßig einnahmen. Ursächlich sind hier sicher Nebenwirkungen zu nennen, wobei die Adhärenz bei $\mathrm{AT}_{1}$-Rezeptorblockern am höchsten lag. Als weitere Ursachen sind eine unzureichende Lebensstilmodifikation mit starkem Alkoholkonsum und Gewichtszunahmen zu nennen. Wie oben dargelegt, muss die parallele Einnahme anderer Medikamente, die zu einer Erschwerung der Blutdruckeinstellung führen können, berücksichtigt werden.

Der erste Schritt in der Evaluation der therapierefraktären Hypertonie ist eine ambulante Langzeitmessung (ABDM) zum Ausschluss einer Pseudoresistenz (Praxishypertonie) sowie die sorgfältige Anamnese des Patienten und der Ausschluss sekundärer Ursachen. In Bezug auf die Adhärenz ist die Umstellung der Medikation auf fixe Kombinationspräparate hilfreich, da diese die Adhärenz der Patienten häufig erhöhen. Eine zweite Möglichkeit beinhaltet die kurze stationäre Aufnahme des Patienten, um die Blutdruckeinstellung unter kontrollierten Bedingungen durchzuführen. Ferner sollte an das Vorliegen eines Schlafapnoesyndroms gedacht werden und ein Schlafapnoe-Screening zur Evaluation durchgeführt werden.

\section{Kurzgefasst}

Bei therapierefraktärer Hypertonie sollte als mögliche Ursache immer auch eine fehlende Adhärenz erwogen werden. Die ABDM kann eine Pseudoresistenz aufdecken. 


\section{Fazit}

$\nabla$

Da erhöhte Blutdruckwerte häufig aufzufinden und mit einem kardiovaskulären Risiko vergesellschaftet sind, sollte zunächst immer eine Basisdiagnostik hinsichtlich bereits vorliegender Endorganschäden mit apparativer Diagnostik, aber auch eine Abklärung eventueller möglicher Ursachen mit Anamnese und Labordiagnostik erfolgen. Bei Verdacht auf eine sekundäre Hypertonie sind vor allem renale, renovaskuläre bzw. endokrinologische Ursachen sowie eine Schlafapnoe auszuschließen.

\section{Zusammenfassung}

$\nabla$

Hochdruckerkrankungen tragen weltweit entscheidend zu Morbidität und Mortalität bei. Nach wie vor ist es für den behandelnden Arzt eine besondere Herausforderung, den Patienten von der Notwendigkeit der Therapie einer häufig asymptomatischen Erkrankung zu überzeugen. Vor der Therapieeinleitung ist eine basisdiagnostische Abklärung auch hinsichtlich des Vorliegens einer sekundären (möglicherweise anders therapeutisch zugänglichen) Hypertonie essenziell. Insbesondere bei jungen Patienten als auch bei fehlender Nachtabsenkung sollte eine ausführliche Abklärung mit Anamneseerhebung und Labordiagnostik sowie Abklärung einer renalen oder endokrinologischen Ursache erfolgen. Weitere Faktoren wie Lebensstil, fehlende Adhärenz, aber auch eine Schlafapnoe sollten in die differenzialdiagnostischen Überlegungen einbezogen werden. Zudem sollte das gesamte kardiovaskuläre Risiko abgeschätzt werden durch Diagnose von anderen Risikofaktoren, Endorganschäden und Begleiterkrankungen.

\section{Abstract}

Arterial hypertension is a major risk factor for morbidity and mortality. The condition is usually asymptomatic and therefore challenging for patient adherence to therapy. The correct diagnostic approach before treatment induction is essential. Especially younger patients and non-dippers require a more detailed diagnostic approach with anamnesis, laboratory parameters and tests for renal or endocrine disorders. In addition, life-style, adherence and sleep apnea should be considered as potential underlying causes for secondary hypertension. Cardiovascular risk should be estimated by diagnosis of risk factors, end-organ damage and concomitant disease.

\section{Interessenkonflikt}

$\nabla$

Keiner der Autoren gibt einen Interessenkonflikt an.

\section{Literatur}

1 Jackson R, Lawes CM, Bennett DA et al. Treatment with drugs to lower blood pressure and blood cholesterol based on an individual's absolute cardiovascular risk. Lancet 2005; 365: 434-441

2 Mancia G, De BG, Dominiczak A et al. 2007 Guidelines for the management of arterial hypertension: The task force for the management of arterial hypertension of the European Society of Hypertension (ESH) and of the European Society of Cardiology (ESC). Eur Heart J 2007; 28: 1462-1536

3 Middeke M. [Blood pressure variability]. Dtsch Med Wochenschr 2011; 136: 2361-2366

4 Luders S, Schrader J, Berger J et al. The PHARAO study: prevention of hypertension with the angiotensin-converting enzyme inhibitor ramipril in patients with high-normal blood pressure: a prospective, randomized, controlled prevention trial of the German Hypertension League. J Hypertens 2008; 26: 1487-1496

5 Franklin SS, Wilkinson IB, McEniery CM. Unusual hypertensive phenotypes: what is their significance? Hypertension 2012; 59: 173-178

6 ALLHAT Officers and Coordinators for the ALLHAT Collaborative Research Group. The Antihypertensive and Lipid-Lowering Treatment to Prevent Heart Attack Trial. Major outcomes in high-risk hypertensive patients randomized to angiotensin-converting enzyme inhibitor or calcium channel blocker vs diuretic: The Antihypertensive and Lipid-Lowering Treatment to Prevent Heart Attack Trial (ALLHAT). JAMA 2002; 288: 2981-2997

7 Adrogue HJ, Madias NE. Sodium and potassium in the pathogenesis of hypertension. N Engl J Med 2007; 356: 1966-1978

8 Puddey IB, Beilin LJ, Rakie V. Alcohol, hypertension and the cardiovascular system: a critical appraisal. Addiction Biol 1997; 2: 159-170

9 Young T, Peppard PE, Gottlieb DJ. Epidemiology of obstructive sleep apnea: a population health perspective. Am J Respir Crit Care Med 2002; 165: $1217-1239$

10 Marin JM, Agusti A, Villar I et al. Association between treated and untreated obstructive sleep apnea and risk of hypertension. JAMA 2012; 307: 2169-2176

11 Mazza A, Zamboni S, Armigliato $M$ et al. Endocrine arterial hypertension: diagnostic approach in clinical practice. Minerva Endocrinol 2008; 33: 127-146

12 Riehl J, Schmitt H, Bongartz D et al. Renal artery stenosis: evaluation with colour duplex ultrasonography. Nephrol Dial Transplant 1997; 12: $1608-1614$

13 Pickering TG, Hall JE, Appel LJ et al. Recommendations for blood pressure measurement in humans and experimental animals: part 1: blood pressure measurement in humans: a statement for professionals from the Subcommittee of Professional and Public Education of the American Heart Association Council on High Blood Pressure Research. Circulation 2005; 111: 697-716 